\title{
Fine-tuning p53 activity through C-terminal modification significantly contributes to HSC homeostasis and mouse radiosensitivity
}

\author{
Yunyuan V. Wang, ${ }^{1}$ Mathias Leblanc, ${ }^{1}$ Norma Fox ${ }^{2}$ Jian-Hua Mao, ${ }^{3,4}$ Kelsey L. Tinkum, ${ }^{5,6,7}$ \\ Kurt Krummel, ${ }^{1}$ Dannielle Engle, ${ }^{1}$ David Piwnica-Worms, ${ }^{6,7,8}$ Helen Piwnica-Worms, ${ }^{5,7,9,10}$ \\ Allan Balmain, ${ }^{3}$ Kenneth Kaushansky, ${ }^{2}$ and Geoffrey M. Wahl ${ }^{1,11}$ \\ ${ }^{1}$ Gene Expression Laboratory, The Salk Institute for Biological Studies, La Jolla, California 92037, USA; ${ }^{2}$ Department of \\ Medicine, University of California at San Diego, La Jolla, California 92093, USA; ${ }^{3}$ The Helen Diller Family Comprehensive \\ Cancer Center, University of California at San Francisco, San Francisco, California 94158, USA; ${ }^{4}$ Life Sciences Division, \\ Lawrence Berkeley National Laboratory, Berkeley, California 94720, USA; ${ }^{5}$ Department of Cell Biology and Physiology, \\ Washington University Medical School, St. Louis, Missouri 63110, USA; ${ }^{6}$ Mallinckrodt Institute of Radiology, Washington \\ University Medical School, St. Louis, Missouri 63110, USA; ${ }^{7}$ The BRIGHT Institute, Washington University Medical School, \\ St. Louis, Missouri 63110, USA; ${ }^{8}$ Department of Developmental Biology, Washington University Medical School, St. Louis, \\ Missouri 63110, USA; ${ }^{9}$ Department of Medicine, Washington University Medical School, St. Louis, Missouri 63110, USA; ${ }^{10}$ the \\ Howard Hughes Medical Institute, St. Louis, Missouri 63130, USA
}

Cell cycle regulation in hematopoietic stem cells (HSCs) is tightly controlled during homeostasis and in response to extrinsic stress. p53, a well-known tumor suppressor and transducer of diverse stress signals, has been implicated in maintaining HSC quiescence and self-renewal. However, the mechanisms that control its activity in HSCs, and how p53 activity contributes to HSC cell cycle control, are poorly understood. Here, we use a genetically engineered mouse to show that $53 \mathrm{C}$-terminal modification is critical for controlling HSC abundance during homeostasis and HSC and progenitor proliferation after irradiation. Preventing p53 C-terminal modification renders mice exquisitely radiosensitive due to defects in $\mathrm{HSC} /$ progenitor proliferation, a critical determinant for restoring hematopoiesis after irradiation. We show that fine-tuning the expression levels of the cyclin-dependent kinase inhibitor p21, a p53 target gene, contributes significantly to p53-mediated effects on the hematopoietic system. These results have implications for understanding cell competition in response to stresses involved in stem cell transplantation, recovery from adverse hematologic effects of DNA-damaging cancer therapies, and development of radioprotection strategies.

[Keywords: p53; C-terminal modification; HSC; radiosensitivity; cell cycle; apoptosis]

Supplemental material is available for this article.

Received December 20, 2010; revised version accepted May 26, 2011.

In its most-studied role, p53 acts as a tumor suppressor that responds to DNA damage, oncogenic, and other cellular stresses to maintain genomic stability (for review, see Toledo and Wahl 2006). However, accumulating evidence reveals other functions for p53 in cell metabolism, the inflammatory response, angiogenesis, reproduction, embryogenesis, and stem cell renewal (Vousden and Prives 2009). These recent observations suggest that p53 may have evolved due to its roles in biological development with tumor suppression being an additional benefit (Aranda-Anzaldo and Dent 2007).

${ }^{11}$ Corresponding author.

E-mail wahl@salk.edu.

Article is online at http://www.genesdev.org/cgi/doi/10.1101/gad.2024411. p53 is critical for maintaining the balance between cell survival and apoptosis during development and homeostasis, often in response to stress. The hematopoietic system is particularly vulnerable to genotoxic stresses such as radiation, which activates p53 and results in dosedependent acute marrow failure due to apoptosis in highly proliferative progenitors and mature blood cells (Gudkov and Komarova 2003). The p53-regulated proapoptotic gene puma is also recently shown to be critical in modulating the function of hematopoietic stem and progenitor cells in response to high-dose irradiation (Shao et al. 2010; Yu et al. 2010). Hematopoietic system restoration relies on the hematopoietic stem cells (HSCs) that reside in the bone marrow (BM). HSCs sustain hematopoiesis through selfrenewal, proliferation, and generation of differentiated 
progeny of distinct blood cell lineages. Radiation-induced damage in the HSC compartment, or forcing HSCs to undergo rapid proliferation, can lead to HSC exhaustion and long-term myelosuppression (Cheng et al. 2000; Wang et al. 2006), which is a common side effect observed in cancer patients receiving chemotherapy and extensive radiation therapy.

Several recent studies using mouse models suggest a critical role for the p53 pathway in HSC self-renewal and quiescence, as p53-null mice exhibit an increased pool of HSCs and their HSCs are more resistant to radiationinduced senescence (TeKippe et al. 2003; Meng et al. 2003; Wang et al. 2006; Chen et al. 2008; Liu et al. 2009). However, precise regulation of p53 activity is likely to be important in determining the response of HSCs and proliferative progenitors to irradiation. Thus, insufficient p53 activation should favor cell survival, but put cells at risk for loss of genomic integrity. In contrast, excessive p53 activation could compromise steady-state hematopoiesis and its recovery following exogenous marrow insult by causing too many cells to be eliminated. While insight has been gained into the impact of p53 itself on radiation sensitivity in the hematopoietic system, such studies have commonly used p53-deficient mice, which are of limited utility as they tend to develop tumors very rapidly (within 3 mo of age) and the heterozygotes quickly succumb to radiation-induced lymphomas (Kemp et al. 1994). This precludes evaluating the mechanisms of radioresistance, which requires dynamic and prolonged observation of radiation effects on hematopoietic cell kinetics. Moreover, p53-null models do not enable analyses of the roles of factors that control p53 level or activity, and these are likely to be critically important. For example, slight increases in p53 activity caused by reduced expression of p53 inhibitors $\mathrm{Mdm} 2$ and $\mathrm{Mdmx}$ render mice more radiosensitive (Mendrysa et al. 2003; Terzian et al. 2007). Conversely, mice encoding a stable form of Mdmx are impressively radioresistant despite modest reduction of p53 activity (Wang et al. 2009). However, whether the differences in radiosensitivity associated with altered p53 activity in these models reside in mature blood cells, the proliferative pool, the HSC compartment, or a combination of these, and the molecular mechanisms by which p53 activation elicits the observed phenotypes, remain to be determined.

In vitro and in vivo studies show that p53 activity is determined to a significant extent by mechanisms that regulate its abundance and stability. $\mathrm{Mdm} 2$ and $\mathrm{Mdmx}$ reduce p53 activity by binding to the $\mathrm{N}$-terminal p53 transactivation domain (TAD) and by promoting ubiquitin-dependent p53 degradation (for review, see Wade et al. 2010). Control of p53 degradation is thought to partially require ubiquitylation of highly conserved C-terminal lysine residues (Rodriguez et al. 2000). The same lysines can also be acetylated by coactivators such as p300 and CBP to promote transactivation of target genes (for review, see Kruse and Gu 2009). In vitro studies suggest a model in which p53 is activated by damage-mediated kinases that induce phosphorylation in $\mathrm{N}$-terminal serines to produce a conformational change leading to $\mathrm{Mdm} 2 / \mathrm{Mdmx}$ dissoci- ation and p300/CBP recruitment (Appella and Anderson 2001). These factors then acetylate the $C$ terminus to stabilize p53 and enhance its transcriptional activity. However, in vivo, these modifications appear to be dispensable during embryogenesis, and do not significantly affect p53 activity in mouse embryonic fibroblasts (MEFs) (Krummel et al. 2005; Feng et al. 2005). This does not address tissue and condition-specific effects of these residues in vivo, nor whether such effects would be manifested through regulation of a subset of p53 target genes. Evidence of the importance of lysine modification for p53 in vivo functions comes from recent studies showing that mutation of K120 impairs puma activation (Tang et al. 2006), and that a K120R/K164R double mutant reduces induction of puma and p21, but not mdm2 (Tang et al. 2008). Thus, lysine modifications in the DNA-binding region appear to play a key role in differential gene regulation.

These observations led us to examine more deeply the impact of modification of the conserved seven C-terminal lysine residues in a knock-in model in which they were replaced with arginine (7KR) to prevent ubiquitylation or acetylation (Krummel et al. 2005). Here, we show that the $p 53^{7 K R}$ mutation plays a critical role in regulating p53 transcriptional activity on a subset of genes in the hematopoietic system. This, in turn, affects maintenance of HSCs during unchallenged homeostatic growth, in the setting of BM transplantation, and after radiation exposure. Notably, the $p 53^{7 K R}$ mutation causes extraordinary radiosensitivity, which is partially rescued by loss of a single p21 allele, implicating C-terminal modification in controlling p21 transcription in the HSC and progenitor pools that play critical roles in radiation responses.

\section{Results}

Wild-type (WT) and $\mathrm{p} 53^{7 \mathrm{KR}}$ mutant mice have similar life spans

We first asked whether $p 53^{7 K R}$ elicits a premature aging phenotype, as reported previously for some mouse mutants with elevated p53 activity (e.g., see García-Cao et al. 2002; Tyner et al. 2002). As such, we followed a large cohort of $p 53^{7 K R}$ and WT animals to ascertain potential aging phenotypes. The data clearly show that the life span is similar between mutant mice and their WT littermates, and that the mutant mice do not display obvious signs of premature aging (Supplemental Fig. S1).

\section{Preventing p53 C-terminal modification engenders exquisite radiosensitivity due to multiple hematopoietic system defects}

To determine whether $p 53^{7 K R}$ mice have altered biological responses to DNA damage, mutant and wild-type (WT) mice were subjected to a range of sublethal doses of whole-body $\gamma$-irradiation $(4,5$, and 6 Gy). As expected, all WT mice survived these exposures. In contrast, $p 53^{7 K R}$ mice in the same genetic background exhibited a striking dose-dependent response to irradiation: Only $\sim 55 \%$ of $p 53^{7 K R}$ mice were alive at $4 \mathrm{wk}$ after $5 \mathrm{~Gy}$ irradiation, while $100 \%$ of $p 53^{7 K R}$ mice died within 4 wk following 6 Gy irradiation (Fig. 1A). 




Figure 1. Preventing C-terminal modification of p53 engenders exquisite radiosensitivity, particularly in the hematopoietic system. (A) Kaplan-Meier radiation survival curves in WT mice and $p 53^{7 K R}$ (7KR) mice after 4, 5, and 6 Gy of whole-body irradiation showing dose-dependent mortality in $p 53^{7 K R}$ mice (4 Gy: $n=58$, $P<0.001$; 5 Gy: $n=25, P<0.001 ; 6$ Gy: $n=10, P<0.001)$. $(B)$ Increased cardiac weights and enlarged hearts in irradiated $p 53^{7 K R}$ mice 4 wk after 5 Gy of irradiation. Error bars represent the SEM from four animals. The representative hearts from nonirradiated and irradiated mice are shown in the picture. $(C)$ $\mathrm{H} \& \mathrm{E}$ staining of thymus, spleen, and $\mathrm{BM}$ from a $p 53^{7 K R}$ mouse that died after exposure to 5 Gy of whole-body irradiation.

After irradiation, the mutant mice exhibited pallor of the carcass, low hematocrit, and enlarged hearts (Fig. 1B). Based on prior studies (Fauci et al. 2008), these observations are consistent with death resulting from heart failure secondary to severe anemia. Indeed, pathological evaluation showed that the radiation-induced lesions in $p 53^{7 K R}$ mice were confined to the hematopoietic organs (Fig. 1C; Supplemental Fig. S2). Importantly, no significant differences were observed between WT and $p 53^{7 K R}$ mice in other organs including radiosensitive tissues such as the gastrointestinal tract (Supplemental Fig. S2; data not shown).

To expand on these results, we compared complete blood counts (CBCs) in WT and $p 53^{7 K R}$ mice prior to and $2 \mathrm{wk}$ following $5 \mathrm{~Gy}$ irradiation. This time point was chosen because myeloablation in mice has been shown to occur within 2 wk of irradiation followed by recovery by $\sim 4$ wk (Uchida et al. 1994). We found that $p 53^{7 K R}$ mice exhibit slightly lower blood counts at baseline (mutant $=80 \%-$ $90 \%$ of WT levels) (Fig. 2A,C). Consistent with previous studies (Wang et al. 2009), we observed a marked decrease in white blood cell (WBC) counts and a mild decrease in red blood cell (RBC) and platelet counts in WT mice $2 \mathrm{wk}$ post-irradiation (Fig. 2B). These changes are p53-dependent, as mild-to-no changes in CBCs occurred in p53-null mice (Fig. 2B). On the other hand, $p 53^{7 K R}$ mice exhibited more severe decreases in all three blood lineages $2 \mathrm{wk}$ post-irradiation (mutant $\sim 30 \%-50 \%$ of WT) (Fig. 2B,C).

Histology of the BM and spleen, the major hematopoietic organs in mice, revealed no visible differences between WT and $p 53^{7 K R}$ mice prior to irradiation (Supplemental Fig. S2). Two weeks after 5 Gy irradiation, WT BM and spleens exhibited moderate to robust intra- and extramedullary hematopoiesis, which gradually abated to near baseline by $4 \mathrm{wk}$ as blood counts returned to normal (Supplemental Fig. S2). $p 53^{7 K R}$ mice exhibited more dramatic changes in hematopoietic organs. As such, some mutants showed robust intra- and extramedullary hematopoiesis in response to the severe radiation-induced pancytopenia, while others displayed severe atrophy of the hematopoietic BM and splenic red pulp (Supplemental Fig. S2). Both observations are consistent with increased radiosensitivity in the hematopoietic system of the $p 53^{7 K R}$ mice. At $4 \mathrm{wk}$, mutant mice showed intra- and extramedullary hematopoiesis, indicative of continued stimulation from persistent pancytopenia. Importantly, hematopoiesis was not sufficient to compensate for the marked pancytopenia in time to ensure survival of half of the mutant mice. Consistent with the survival curve (Fig. 1A), 6 Gy irradiation translated histopathologically as a more severe atrophy in the BM and spleen (Supplemental Fig. S2).

The peripheral pancytopenia observed in irradiated $p 53^{7 K R}$ mice could reflect impaired hematopoietic stem and progenitor cell survival, proliferation, or differentiation defects, increased blood cell destruction, or a combination of these. Importantly, responses measured by peripheral blood cell counts can underestimate a progenitor cell phenotype, as the post-progenitor cell hematopoietic compartment can hyperproliferate to compensate for profound deficiencies in marrow stem or progenitor cells (Kaushansky et al. 2002). As such, we evaluated total $\mathrm{BM}$ counts before and after $5 \mathrm{~Gy}$ irradiation to further understand the factors involved in the increased radiosensitivity of $p 53^{7 K R}$ mice. The total BM counts in WT and $p 53^{7 K R}$ mice were not significantly different prior to irradiation (Fig. 2D). Consistent with previous studies (Wang et al. 2006), the total BM cells from WT mice dropped significantly by $4 \mathrm{~d}$ after irradiation, and then started to recover by 2 wk post-irradiation. Four weeks after irradiation, total $\mathrm{BM}$ cells increased to $\sim 85 \%$ of baseline levels. Importantly, total BM cells in $p 53^{7 K R}$ mice decreased to similar levels as WT at $4 \mathrm{~d}$ but remained significantly lower at 2 and 4 wk post-irradiation (Fig. 2D).

We further quantified hematopoietic progenitor cells by culturing marrow cells from WT and $p 53^{7 K R}$ mice in semisolid medium containing various growth factor combinations. Figure $2 \mathrm{E}$ shows that, compared with WT animals, $p 53^{7 K R}$ mouse marrow may contain slightly fewer granulocytic/macrophage (CFU-GM), erythroid (BFU-E), and megakaryocytic (CFU-MK) progenitor cells prior to irradiation (differences are not statistically significant). Immediately following irradiation and isolation of 

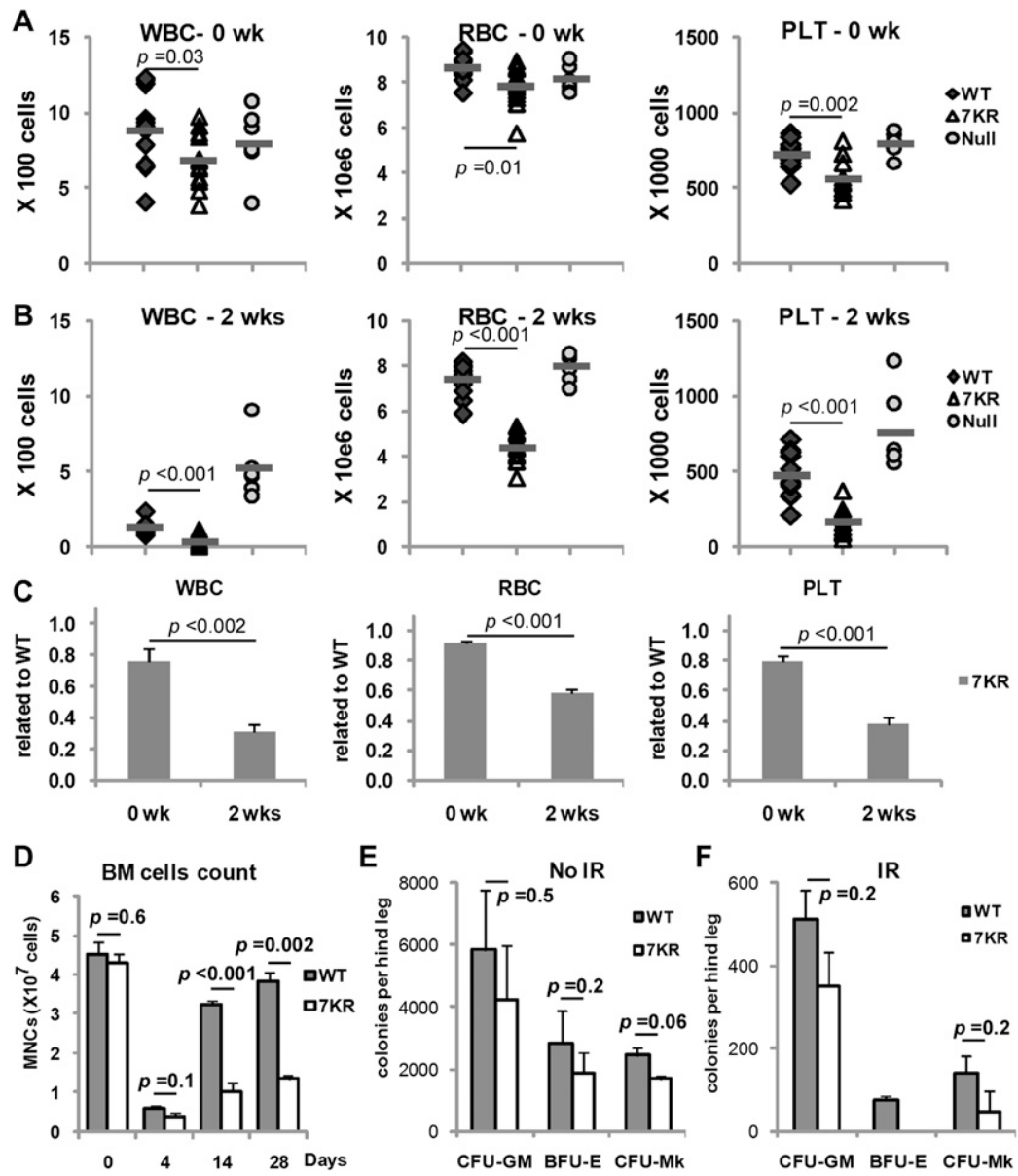

Figure 2. Irradiation causes long-term defects in $p 53^{7 K R}$ hematopoietic progenitor cells. $(A)$ White blood cells (WBC) red blood cells (RBC), and and platelets (PLT) in WT, $p 53^{7 K R}$, and $p 53-$ null mice prior to irradiation. WT and $7 K R: n=$ 13; null: $n=6$. $(B)$ Blood cell counts at $2 \mathrm{wk}$ after 5 Gy of whole-body irradiation. $(C)$ The fold changes of blood cell counts in $p 53^{7 K R}$ mice related to WT mice 2 wk post-irradiation. $(D)$ Mononucleated cell (MNCs) count in BM at baseline and 4, 14, and $28 \mathrm{~d}$ post-irradiation revealed insufficient recovery of BM cells in irradiated $p 53^{7 K R}$ mice. $(E)$ BM cells were isolated from nonirradiated mice and seeded for granulocytic/macrophage (CFU-GM), erythroid (BFU-E), and megakaryocytic (CFU-MK) progenitor cell colony-forming assay. (F) BM cells were immediately isolated from animals that were exposed to irradiation for progenitor colony-forming assay. Note: No colonies were found when $5 \times 10^{6}$ $p 53^{7 K R} \mathrm{BM}$ cells were seeded for BFU-E assay. The error bars in $D, E$, and $F$ represent SEM from three independent experiments. marrow cells, we observed a 10 - to 20 -fold reduction of colony-forming cells of all cell types in both WT and $p 53^{7 K R}$ mice, again with slightly fewer colony-forming cells in $p 53^{7 K R}$ mice (Fig. 2F). In contrast, and consistent with lower total BM cell counts at 2 and 4 wk postirradiation (Fig. 2D), the mutant mice exhibited more severe reductions in hematopoietic colony-forming progenitor cells when marrows were isolated 2 and 4 wk postirradiation (Supplemental Fig. S3).

p53 ${ }^{7 \mathrm{KR}}$ BM cells compete poorly with WT cells in transplantation and are more radiosensitive

The radiosensitivity observed in the colony formation assays implies a defect in the progenitor and/or stem cell compartments. We therefore used competitive BM transplantation to address whether the long-term stem cell repopulating abilities of the WT and mutant differ. BM cells from donor mice (WT or $p 53^{7 K R}$ ) expressing the CD45.2 leukocyte cell surface marker were transplanted into lethally irradiated recipient mice in fixed ratios with WT competitor BM cells expressing the CD45.1 leukocyte cell surface marker (Fig. 3A). Twelve weeks after transplantation, at which time only long-term HSCs (LT-HSCs) contribute to hematopoiesis, mice were sacrificed and leukocytes from peripheral blood and BM were analyzed for expression of CD45.1 and CD45.2 using flow cytometry. As expected, WT donor cells (CD45.2) and competitor cells (CD45.1) transplanted in equal amounts $\left(2 \times 10^{5}\right)$ into lethally irradiated recipients competed almost equally to repopulate the peripheral blood compartment (Fig. 3C). In contrast, under the same experimental conditions, $p 53^{7 K R}$ CD45.2 donor cells only contributed to $1.2 \%$ of the reconstituted peripheral blood cell populations at $12 \mathrm{wk}$ post-transplantation.

We further characterized the radiation-induced defect in $p 53^{7 K R}$ HSCs by performing the same competitive repopulation assays with irradiated WT and $p 53^{7 K R}$ cells. BM cells were isolated $4 \mathrm{~d}$ post-irradiation, as this corresponds to the time of maximal radiation-induced BM cell lethality (Wang et al. 2006). Since irradiation significantly affects multiple hematopoietic cell types and can induce HSC senescence (Meng et al. 2003), we used three or 10 times more irradiated WT or $p 53^{7 K R}$ cells than nonirradiated competitor cells to ensure the presence of some viable HSCs from irradiated donors for engraftment. Under these experimental conditions, irradiation of WT donor cells produced a 10to 15 -fold reduction in transplantation efficiency as compared with nonirradiated WT donor cells (Fig. 3C).

Identical analysis using $p 53^{7 K R}$ donor cells revealed such a profound defect in $p 53^{7 K R}$ HSCs that none of the reconstituting cells were derived from the mutant 


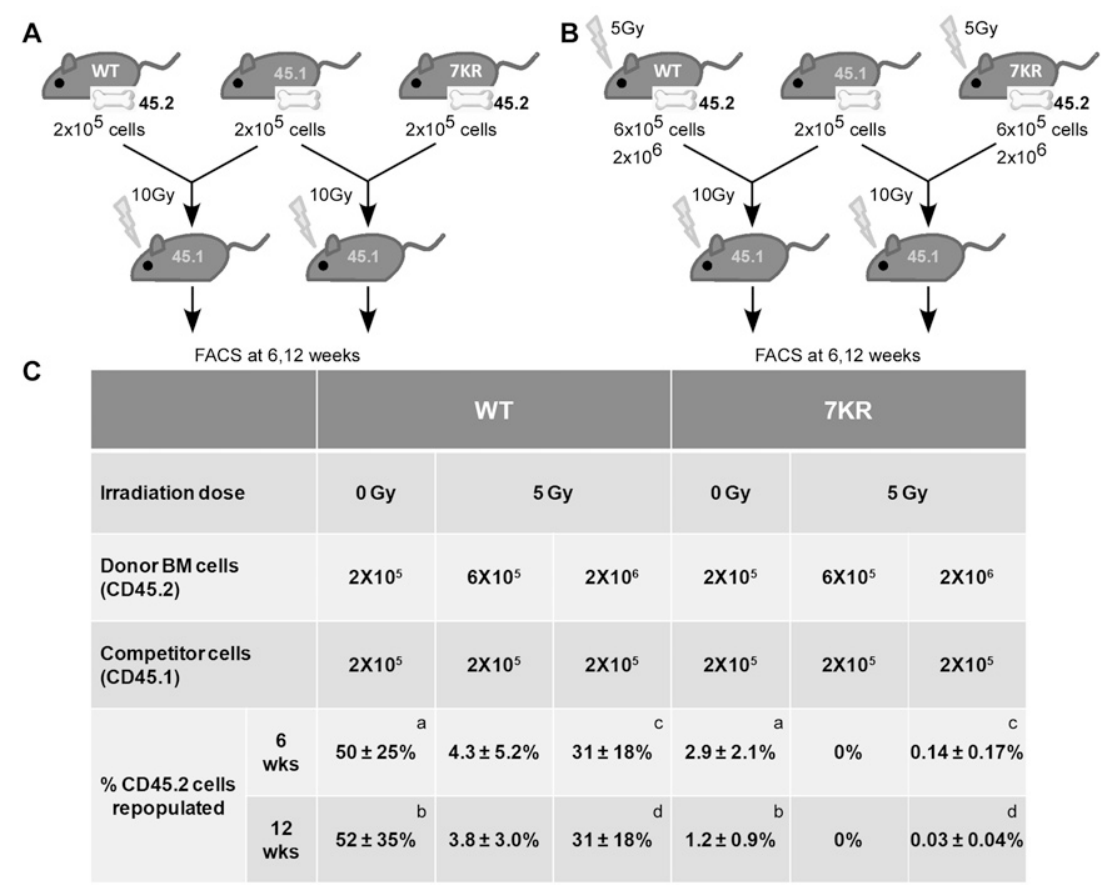

Figure 3. $p 53^{7 K R}$ HSCs exhibit sensitivity to transplantation and to irradiation. BM from nonirradiated $(A)$ and 4-d post-irradiated $(B)$ WT or $p 53^{7 K R}$ donor mice expressing the CD45.2 leukocyte cell surface marker were transplanted into lethally irradiated recipient mice in fixed ratios with WT competitor marrow cells expressing CD45.1. (C) $p 53^{7 K R}$ HSCs did not compete as well as WT for longterm repopulation under both nonirradiated and irradiated conditions. At $6 \mathrm{wk}$ and $12 \mathrm{wk}$ post-transplantation, peripheral blood was withdrawn from the recipients and analyzed for the percentage of CD45.2 cells in total leukocytes. Results show mean \pm SD from at least five animals. a, b: $P<0.05$. c, d: $P<$ 0.001 . animals when threefold more irradiated $p 53^{7 K R}$ cells were cotransplanted with competitor WT cells. Even with 10fold more irradiated $p 53^{7 K R}$ donor cells, mutant HSCs were not capable of competing in the reconstitution of the hematopoietic compartment (Fig. 3C). These data reveal a profound defect in the ability of $p 53^{7 K R}$ HSCs to compete with WT HSCs for repopulation, and that this phenotype is magnified by irradiation.

\section{Unchallenged $\mathrm{p} 53^{7 \mathrm{KR}}$ BM contains fewer HSCs}

In addition to a reduced competitive fitness of the mutant HSCs, we also explored the possibility that nonirradiated $p 53^{7 K R}$ BM contains fewer HSCs. We estimated HSC numbers by enumerating $\mathrm{Lin}^{-} \mathrm{Scall}^{+} \mathrm{cKit}^{+}$(LSK) cells, a population of cells previously shown to be highly enriched for HSCs (Wognum et al. 2003). We observed that $p 53^{7 K R} \mathrm{BM}$ contains approximately two-thirds the number of LSK cells as compared with WT BM (Fig. 4A; Supplemental Fig. S4). We confirmed this result by sorting for an independent set of HSC cell surface markers, referred to as SLAM markers $\left(\mathrm{Lin}^{-} \mathrm{CD} 41^{-} \mathrm{CD} 48^{-} \mathrm{CD} 150^{+}\right)$, as this yields a population that contains $40 \%-50 \%$ HSCs as determined by limiting dilution long-term repopulation analysis (Kiel et al. 2005). As observed for LSK cells, SLAM cells from $p 53^{7 K R}$ marrows were $\sim 40 \%$ the abundance of similarly isolated cells from WT BM (Fig. 4B; Supplemental Fig. S4).

The radiosensitivity of $\mathrm{p} 53^{7 \mathrm{KR}}$ animals resides in the HSC and not in the stem cell niche

We next determined whether irradiation induces changes in the HSC niche(s) in $p 53^{7 K R}$ animals, as this could also contribute to their reduced abundance and to their radio- sensitivity. We investigated this possibility by transplanting $1 \times 10^{6} \mathrm{WT}(\mathrm{CD} 45.1) \mathrm{BM}$ cells into either WT or $p 53^{7 K R}$ mice exposed to the lethal dose of 10 Gy whole-body radiation (Fig. 4B). We observed that $100 \%$ of these lethally irradiated $p 53^{7 K R}$ mice survived after transplantation of WT BM. FACS analyses showed that donor cells (CD45.1) reconstituted the entire hematopoietic system by $6 \mathrm{wk}$ post-transplantation (Supplemental Fig. S5). The rescued mice are thus chimeric in that their hematopoietic compartment is fully reconstituted by WT HSCs, while the marrow niche and all other cells in these animals are $p 53^{7 K R}$. CBC analysis showed that these WT: $p 53^{7 K R}$ chimeras resisted 5 Gy of whole-body irradiation like WT:WT chimaeras (Fig. 4C). This restoration of radioresistance was not due to an effect of the age of the animals at the time of analysis, as 23-wk-old $p 53^{7 K R}$ mice (slightly older than the irradiated chimeras) were still sensitive to the same dose of irradiation (Supplemental Fig. S6). This result demonstrates that introducing WT HSCs by BM transplantation is sufficient to rescue the radiosensitivity of $p 53^{7 K R}$ animals.

\section{$\mathrm{p} 53^{7 \mathrm{KR}}$ function and radiosensitivity are affected by $M d m 2$}

Previous studies of the impact of $M d m 2$ and $M d m x$ expression on $\mathrm{p} 53$ activity revealed that the p53 pathway is exquisitely sensitive to p53 protein levels and basal activity, which can be modulated by p53-negative regulators (Mendrysa et al. 2003; Terzian et al. 2007; Wang et al. 2009). Since $M d m 2$ heterozygous mice are known to have elevated p53 activity (Terzian et al. 2007), we compared their radiosensitivity with $p 53^{7 K R}$ mice. Interestingly, while half of $p 53^{7 K R}$ mice died from 5 Gy ionizing 


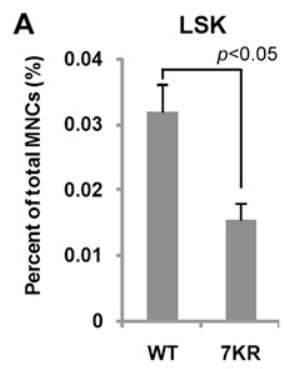

C
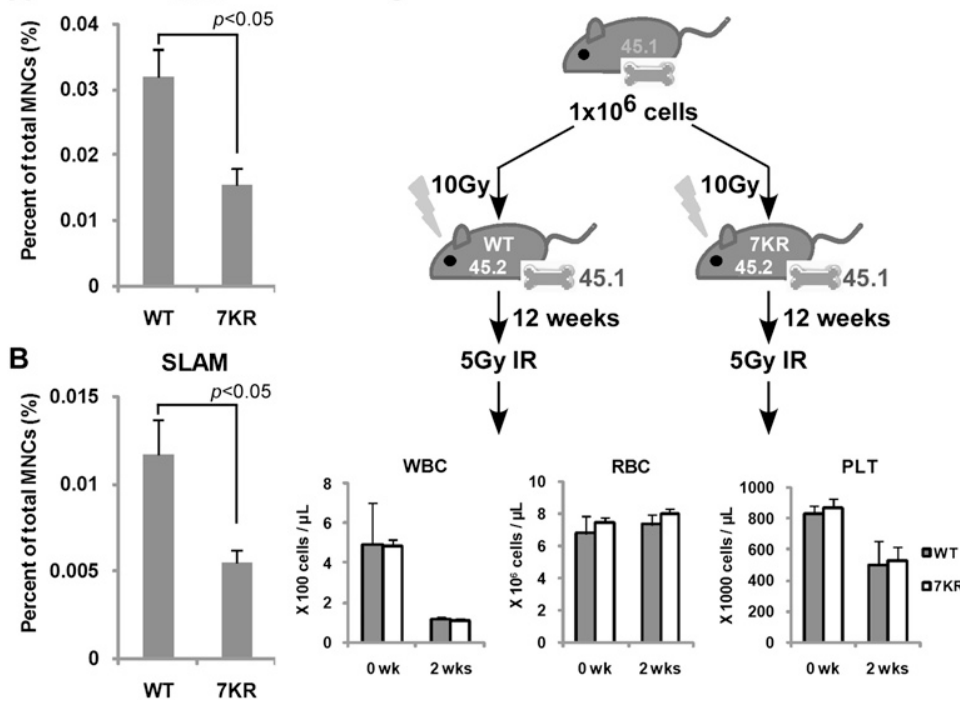

Figure 4. The hematopoietic defects in $p 53^{7 K R}$ mice reside in HSCs/progenitor cells and not in the stem cell niche. (A) BM cells were isolated from nonirradiated mice and stained with antibodies against hematopoietic lineage (Lin) markers and cell surface marks Scal and c-Kit and analyzed by flow cytometry. $\mathrm{Lin}^{-} \mathrm{Scal}^{+} \mathrm{c}-\mathrm{Kit}^{+}$ subpopulation were gated as HSC-enriched LSK cells. Cell plots are shown in Supplemental Figure S4. (B) Antibody cocktail lineage markers CD41, CD48, and CD150 were used for the analysis. The $\mathrm{Lin}^{-} \mathrm{CD} 41^{-} \mathrm{CD} 48^{-} \mathrm{CD} 150^{+}$subpopulation was gated as HSC-enriched SLAM cells. Cell plots are shown in Supplemental Figure S4. $(C)$ Introducing WT HSCs into $p 53^{7 K R}$ mice by $\mathrm{BM}$ transplantation overcame the radiosensitivity of the animals. BM cells expressing CD45.1 were transplanted into lethally irradiated WT or $p 53^{7 K R}$ mice. Twelve weeks later, the chimeric mice were exposed to $5 \mathrm{~Gy}$ of irradiation followed by $\mathrm{CBC}$ analysis. $(A-C)$ Error bars represent the SEM from three animals. radiation (IR), all $M d m 2^{+/-}$mice survived. Moreover, loss of one $M d m 2$ allele in a $p 53^{7 K R}$ background significantly increased $p 53^{7 K R}$ radiosensitivity (Fig. 5A). CBC and histopathological evaluation revealed that $p 53^{7 K R} ; M d m 2^{+/-}$ mice exhibited a more severe radiation-induced pancytopenia concomitant with severe atrophy in the spleen and BM (Fig. 5B,C). $p 53^{7 K R} ; M d m 2^{+/-}$BM also had slightly fewer HSC-enriched SLAM cells at baseline when compared with $p 53^{7 K R} ; M d m 2^{+/+}$littermates (Fig. 5E). It is unlikely that genetic background differences underlie any of these effects, as CBC analyses showed similar responses in the backcrossed $p 53^{7 K R}$ and $p 53^{7 K R} ; M d m 2^{+/+}$ genotypes (Supplemental Fig. S7).

We next asked whether the relative radiosensitivity of the various genotypes analyzed above correlated with basal p53 protein levels. Surprisingly, the basal levels of p53 in splenocytes from the different genotypes (Fig. 5D) showed no direct correlation with the severity of the radiation response. At baseline, p53 level was slightly higher in $p 53^{7 K R}$ mice than in WT or $M d m 2^{+/-}$animals, while it was, if anything, slightly lower in the more radiosensitive $p 53^{7 K R} ; M d m 2^{+/-}$mice (Fig. 5D; Supplemental Fig. S8). In contrast, we observed a significant increase in basal p21 protein levels in $p 53^{7 K R}$ mice, and this was substantially stronger in $p 53^{7 K R} ; M d m 2^{+/-}$mice. Furthermore, the $\mathrm{p} 21$ level in the $p 53^{7 K R} ; M d m 2^{+/-}$mice was the highest of any genotype after irradiation, and this correlates with their exquisite radiosensitivity. Thus, the p21 levels parallel the relative radiosensitivity of each genotype analyzed. This raises the possibility that the $\mathrm{p} 53^{7 \mathrm{KR}}$ mutant activates a different transcriptional program than $\mathrm{p} 53^{\mathrm{WT}}$, and that this program may be further modulated by changes in Mdm2 levels.

\section{p53 $3^{7 \mathrm{KR}}$ exhibits differential transcriptional activity}

Recent studies show that post-translational modifications of different lysine residues in p53 can elicit differential effects on gene regulation that have significant biological consequences (Tang et al. 2008; W Gu, pers. comm.). Therefore, we investigated whether the K-to-R mutations we introduced into p53 C-terminal lysines also elicited differential effects on target gene transcription that contribute to the radiosensitivity of the mutant animals. As a first analysis, quantitative PCR (qPCR) measurements revealed that the induced levels of proapoptotic puma and noxa in the thymus and BM were very similar in WT and $p 53^{7 K R}$ after 5 Gy of whole-body irradiation, (Fig. 6A; data not shown). Consistent with our previous studies (Krummel et al. 2005), we found that p21 expression was slightly higher in irradiated $p 53^{7 K R}$ thymus compared with WT thymus (Fig. 6B; Supplemental Fig. S9). Furthermore, p21 was expressed at even higher levels in BM than in thymus, and is reproducibly expressed at twofold higher levels in the $p 53^{7 K R} \mathrm{BM}$ than in WT BM at baseline and after irradiation (Fig. 6B; Supplemental Fig. S9).

We further studied the transcriptional response elicited by $\mathrm{p} 53$ in BM and thymus in the different genotypes using a microfluidic chip that enables qPCR analysis of 43 p53 target genes. This provided a greater sampling of genes to explore and quantify genotype- and tissue-related differences in transcriptional activity. Cluster analysis allowed us to identify gene clusters that were expressed differentially in tissues and/or in response to irradiation (Fig. 6C). For example, cluster IV contains a subset of genes that were highly induced by irradiation in the BM, while cluster II contains genes that were highly induced in the thymus. We noted that several proapoptotic genes lapaf1, bax, puma, and noxa) are present in cluster II. Importantly, these genes were induced to similar levels in WT and $p 53^{7 K R}$ animals. This result suggested that apoptosis might be induced to similar high levels in the WT and mutant animals in the thymus, and to similar but lower levels in the BM. We investigated this directly by terminal deoxynucleotidyl transferase (TdT)-mediated dUTP nick endlabeling (TUNEL) assay. Figure 6D shows that cells in the 

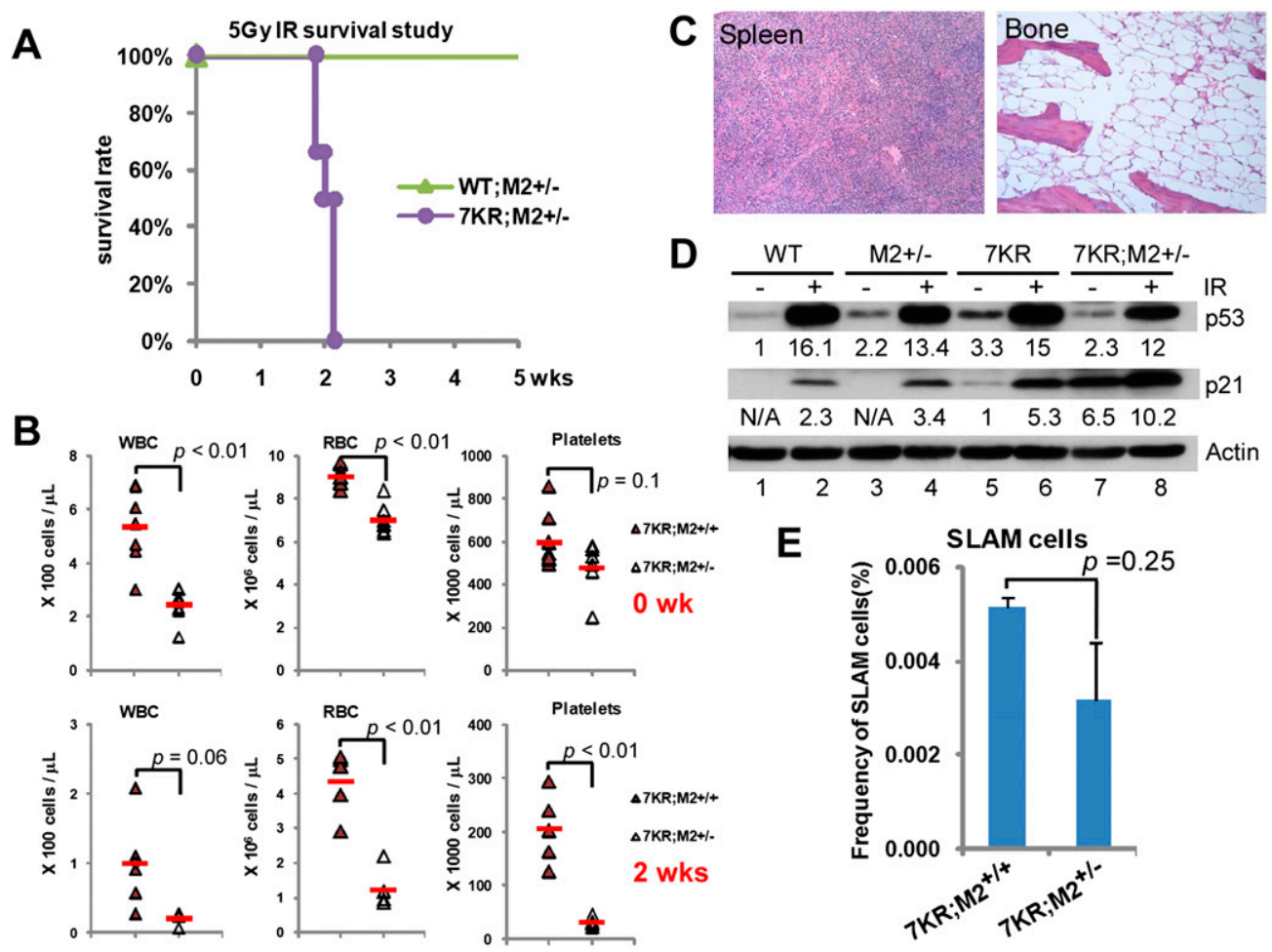

Figure 5. $\mathrm{Mdm} 2$ dependence of radiosensitivity in $p 53^{7 K R}$ mice. $(A)$ Kaplan-Meier radiation survival curves. WT; $M d m 2^{+/-}(n=9)$ and $p 53^{7 K R} ; M d m 2^{+--}(n=6)$ mice under the same genetic background were exposed to $5 \mathrm{~Gy}$ whole-body irradiation. Losing one allele of $M d m 2$ enhanced the radiosensitivity of $p 53^{7 K R}$ mice. $P<0.001$. (B) CBC analysis of $p 53^{7 K R} ; M d m 2^{+/+}$and $p 53^{7 K R} ; M d m 2^{+/-}$mice before irradiation $(n=9)$ and 2 wk after 5 Gy whole-body irradiation $(n=5)$ showed severe pancytopenia in $p 53^{7 K R} ; M d m 2^{+/-}$mice. $(C)$ Histopathological evaluation revealed that $p 53^{7 K R} ; M d m 2^{+/-}$mice that died from irradiation exhibited severe atrophy in the spleen and BM. $(D)$ Western analysis of p53 and $\mathrm{p} 21$ abundance in spleens isolated from WT, $M d m 2^{+/-}(\mathrm{M} 2+/-), p 53^{7 K R}(7 \mathrm{KR})$, and $p 53^{7 K R} ; M d m 2^{+/-}(7 K R ; M 2+/-)$ mice prior to or $3 \mathrm{~h}$ after $5 \mathrm{~Gy}$ of whole-body irradiation. Actin blot provides a loading control. Intensities of p53 and p21 signals were normalized to Actin signals and are presented as relative fold differences. $(E) p 53^{7 K R} ; M d m 2^{+/-}$mice exhibited a slightly lower frequency of HSC-enriched SLAM cells when compared with $p 53^{7 K R} ; M d m 2^{+/+}$mice. Error bars represent the SEM from three animals.

thymus and spleen start to undergo apoptosis at $3 \mathrm{~h}$ postirradiation and that the number of apoptotic cells increases by $6 \mathrm{~h}$. In contrast, few BM cells were apoptotic at this time point. More importantly, there was no measurable difference in apoptotic frequency of cells in WT and $p 53^{7 K R}$ mice (Supplemental Fig. S10).

In addition to the cluster analysis, we identified several genes (Fig. 6C, highlighted in pink) whose expression was strongly and differentially affected by the 7KR mutations. Consistent with the above analyses, we observed higher $p 21$ expression in irradiated $p 53^{7 K R}$ thymus than in irradiated WT thymus (Fig. 6C; Supplemental Fig. S11) and even higher $p 21$ levels in irradiated $p 53^{7 K R} \mathrm{BM}$ (Fig. $6 \mathrm{C}$; Supplemental Fig. S11). Indeed, these analyses revealed $p 21$ to be one of the most significantly upregulated p53-inducible genes we analyzed in irradiated $p 53^{7 K R} \mathrm{BM}$.

We next determined whether the significant differences in $p 21$ expression correlated with IR-induced proliferation differences between WT and $p 53^{7 K R} \mathrm{BM}$ cells. BrdU was administered to mice for $2 \mathrm{~d}$, marrow was isolated, and cells of different hematopoietic lineages were analyzed for BrdU incorporation by flow cytometry. Interestingly, we observed a slightly higher number of
BrdU-positive $\left(\mathrm{BrdU}^{+}\right)$total BM-MNCs (BM mononucleated cells) in $p 53^{7 K R}$ mice relative to WT mice at baseline (Fig. 6E). However, the number of BrdU ${ }^{+}$LSK cells, and the more quiescent HSC-enriched LSKCD34- subpopulation, was lower in $p 53^{7 K R}$ than in WT mice. Upon irradiation, the number of $\mathrm{BrdU}^{+} \mathrm{Lin}^{-}$cells, BrdU $\mathrm{BSK}^{+}$ cells, and $\mathrm{BrdU}{ }^{+} \mathrm{LSKCD}^{-} 4^{-}$cells were significantly lower in $p 53^{7 K R}$ mice than in WT mice (Fig. 6E). Taken together, the data reveal differences in the hematopoietic cell cycle kinetics in unirradiated $p 53^{7 K R}$ and WT animals, with the mutant manifesting greater sensitivity to radiation-induced withdrawal from the cell cycle.

\section{$\mathrm{p} 21$ gene dosage affects radiosensitivity of $\mathrm{p} 53^{7 \mathrm{KR}}$ mice}

Several lines of evidence indicate that puma-mediated apoptosis and $p 21$-mediated cell cycle arrest contribute to HSC abundance and functionality after irradiation (Cheng et al. 2000; Meng et al. 2003; Shao et al. 2010; Yu et al. 2010). While WT and $p 53^{7 K R}$ mice exhibited similar levels of puma induction (Fig. 6B,C) and apoptosis at early times after irradiation (Figs. 2D, 6D; Supplemental Fig. S10), p21 was significantly and differentially expressed in $p 53^{7 K R} \mathrm{BM}$ cells. We determined whether the increased $\mathrm{p} 21$ induction 
A

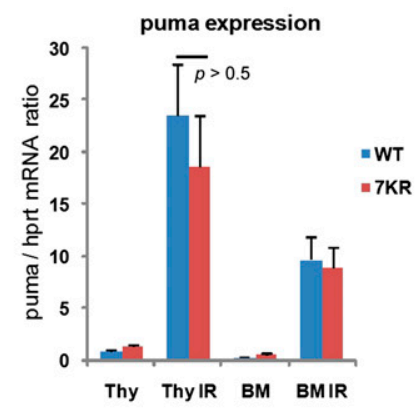

B

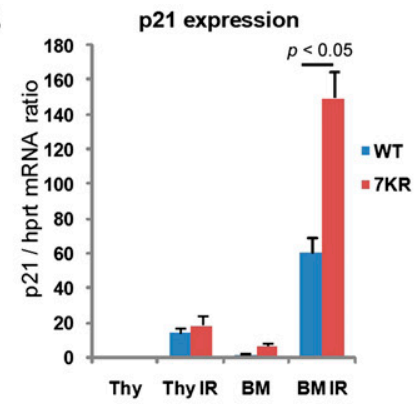

C
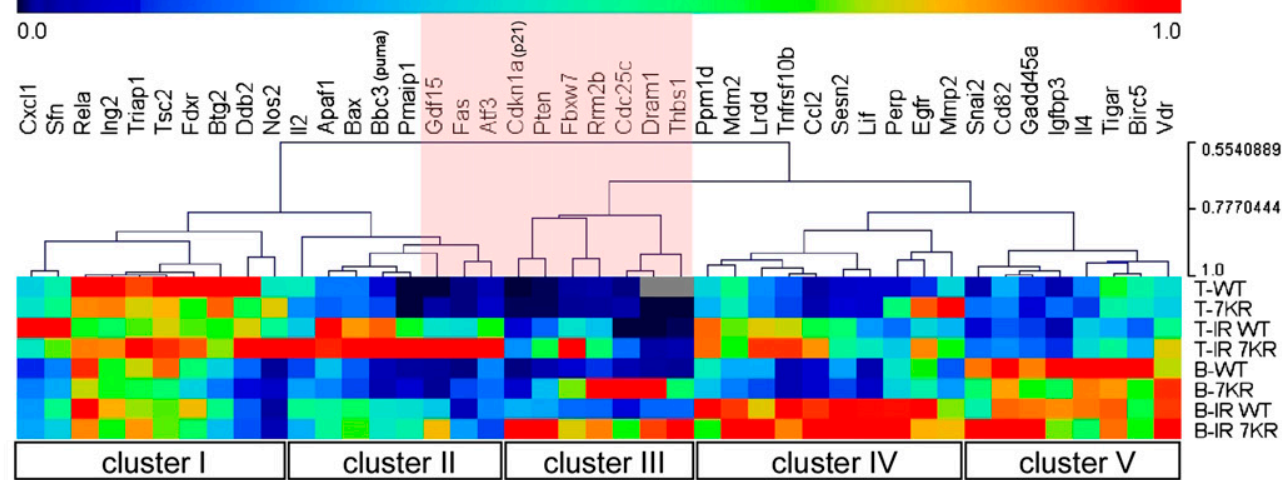

D

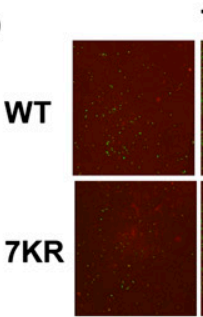

oh

Thymus

Spleen

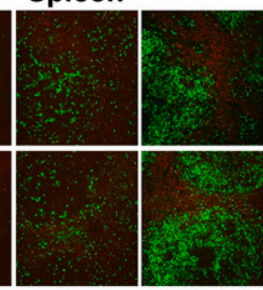

3h

$6 h$

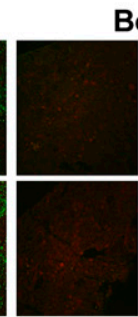

Oh

Bone marrow

E

\begin{tabular}{|c|c|c|c|c|c|}
\hline Genotype & $\mathbf{W T}^{\mathrm{a}}$ & $7 K^{b}$ & WT-IR c & 7KR-IR d & \\
\hline Total MNCs gated $\left(\mathrm{X} 10^{5}\right)$ & $51.12 \pm 2.74$ & $52.19 \pm 2.14$ & $6.41 \pm 0.32$ & $6.41 \pm 0.29$ & \\
\hline $\mathrm{BrdU}+\mathrm{MNCs}\left(\mathrm{X} 10^{5}\right)$ & $32.47 \pm 1.68$ & $38.30 \pm 3.15$ & $2.44 \pm 0.54$ & $3.07 \pm 0.59$ & \\
\hline$\%$ BrdU+MNCs & $63.53 \%$ & $73.38 \%$ & $38.06 \%$ & $47.83 \%$ & \\
\hline BrdU+ Lin- cells $\left(\mathrm{X} 10^{3}\right)$ & $244.93 \pm 13.86$ & $207.34 \pm 9.70$ & $3.81 \pm 0.10$ & $1.66 \pm 0.58$ & \\
\hline$\%$ BrdU+Lin- cells & $4.79 \%$ & $3.97 \%$ & $0.594 \%$ & $0.259 \%$ & $c, d p<0.05$ \\
\hline BrdU+ LSK cells & $1135 \pm 326$ & $806 \pm 319$ & $517 \pm 103$ & $152 \pm 67$ & \\
\hline$\%$ BrdU+LSK cells & $0.022 \%$ & $0.015 \%$ & $0.081 \%$ & $0.024 \%$ & c,d $p<0.05$ \\
\hline BrdU+ LSKCD34- cells & $87 \pm 15$ & $51 \pm 29$ & $22 \pm 2$ & $7 \pm 2$ & \\
\hline$\%$ BrdU+LSK CD34-cells & $0.0017 \%$ & $0.0010 \%$ & $0.0034 \%$ & $0.0011 \%$ & ${ }^{c, d} p<0.05$ \\
\hline
\end{tabular}

Figure 6. Differential gene expression and cell cycle kinetics in $p 53^{7 K R}$ mice. $(A)$ The p53-inducible proapoptotic gene puma was expressed at higher levels in thymus than in BM after irradiation. The level of puma expression was similar between WT and $p 53^{7 K R}$ mice. (B) The p53-inducible cell cycle arrest gene $p 21$ was highly expressed in BM after irradiation, and showed even greater expression in $p 53^{7 K R}$ animals. $(A, B)$ Error bars represent the SEM from three animals. $(C)$ Expression of 43 p53 target genes from thymus $(T)$ and BM $(B)$ prior to irradiation or $3 \mathrm{~h}$ post-irradiation (IR) was detected using microfluidic qPCR analysis. Relative expressions of each gene from all samples were scaled from 0 to 1, followed by hierarchical clustering analysis. (Cluster I) Repressive or less responsive genes. (Cluster II) Highly IR-induced genes in thymus. (Cluster III) Genes highly affected by 7KR mutations in BM. (Cluster IV) Highly IR-induced genes in BM. (Cluster V) Highly expressed genes in BM. The analysis suggests that several genes (highlighted in pink) are differentially affected by the 7KR mutations. $(D)$ Thymi, spleens, and BM were isolated from mice at 0,3 , and $6 \mathrm{~h}$ after 5 Gy of whole-body irradiation. Apoptosis was detected by TUNEL staining (green). (E) Nonirradiated mice or mice exposed to 5 Gy of irradiation were injected with 2 mg of BrdU followed by 2-d administration of BrdU water $(1 \mathrm{mg} / \mathrm{mL})$. BMs were then isolated and stained with antibodies against lineage (Lin) markers; cell surface markers Scal, c-kit, and CD34; and BrdU, followed by flow analysis. BrdU incorporated cell in Lin ${ }^{-}$, LSK $\left(\mathrm{Lin}^{-} \mathrm{Sca} 1^{+} \mathrm{c}-\mathrm{kit}^{+}\right)$and LSKCD34- ${ }^{-}$subcell populations were analyzed. Error bars represent the SD from three animals. 
was functionally relevant to the radiosensitivity of $p 53^{7 K R}$ mice by determining whether reducing p21 gene dosage would reduce the $p 53^{7 K R}$ radiosensitivity. We crossed $p 53^{7 K R}$ mice with $p 21^{+/-}$mice to obtain $p 53^{7 K R} ; p 21^{+/-}$ mice, and found a significant increase in radioresistance in these mice. For example, we observed no mortality in $p 53^{7 K R} ; p 21^{+/-}$mice at least $6 \mathrm{wk}$ after exposure to $5 \mathrm{~Gy}$ radiation (Supplemental Fig. S12). When mice were exposed to $6 \mathrm{~Gy}$ radiation, almost $100 \%$ of $p 53^{7 K R} ; p 21^{+/+}$ mice died within 4 wk of irradiation, while $50 \%$ of $p 53^{7 K R} ; p 21^{+/-}$littermates survived during the same time period. By 8 wk after irradiation, $30 \%$ of $p 53^{7 K R} ; p 21^{+/-}$ mice were still alive (Fig. 7A). Western blot analysis and gene expression confirmed that p21 levels were lower in irradiated $p 53^{7 K R} ; p 21^{+/-}$mice relative to irradiated $p 53^{7 K R} ; p 21^{+/+}$mice (Fig. 7B,C). When HSC-enriched SLAM
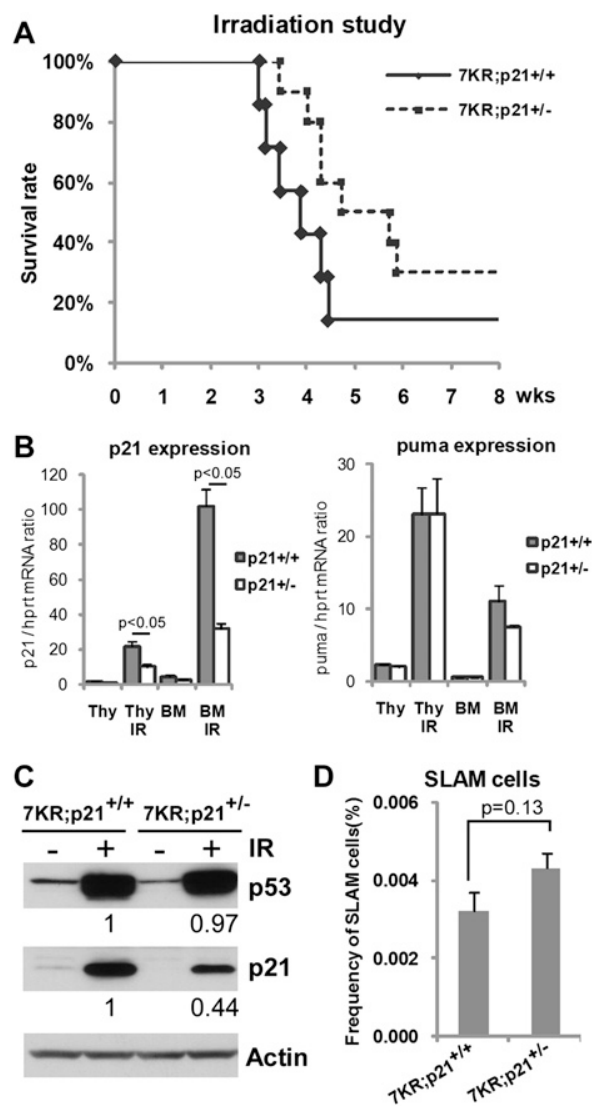

Figure 7. $p 21$ gene dosage affects radiosensitivity of $p 53^{7 K R}$ mice. $(A)$ Kaplan-Meier radiation survival curves. Littermate $p 53^{7 K R} ; p 21^{+/+}(n=7)$ and $p 53^{7 K R} ; p 21^{+/-}(n=10)$ mice were exposed to $6 \mathrm{~Gy}$ of whole-body irradiation. Losing one allele of $p 21$ partially rescued the sensitivity of $p 53^{7 K R}$ mice to $6 \mathrm{~Gy}$ irradiation. $P=0.06$. $(B)$ Analysis of gene expression in thymus and $\mathrm{BM}$ showed that $p 21$ expression was reduced by half in $p 53^{7 K R} ; p 21^{+-}$mice $\left(\mathrm{p} 21^{+/-}\right)$, while puma expression was not affected. Error bars represent the SEM from three animals. $(C)$ Western analysis showed reduced p21 levels in irradiated $p 53^{7 K R} ; p 21^{+/-}$mice, while p53 levels were unchanged. (D) $p 53^{7 K R} ; p 21^{+/-}$mice exhibited slight lower frequency of HSCenriched SLAM cells when compared with $p 53^{7 K R} ; p 21^{+/+}$mice. Error bars represent the SEM from three animals. cells were analyzed, we observed slightly more SLAM cells in $p 53^{7 K R} ; p 21^{+/-}$BM (Fig. 7D), although the increase did not achieve statistical significance. Together, our data demonstrate the importance of proper C-terminal p53 modification to ensure that the p53-dependent cell cycle arrest function is appropriately regulated. Furthermore, they reveal the importance of properly regulated p53induced cell cycle arrest for hematopoietic system recovery after genotoxic stress. They also indicate that p21 contributes to, but is likely not the only factor responsible for, the increased radiosensitivity of $p 53^{7 K R}$ mice.

\section{Discussion}

Our data provide the first evidence that post-translational modifications at highly conserved lysines in the p53 C-terminal regulatory domain are critically important for fine-tuning p53 activity to properly maintain hematopoietic system homeostasis and enable appropriate responses to DNA damage. The inability to post-translationally modify these sites leads to altered expression of a subset of genes that prevents the timely re-entry of HSCs and progenitors into the cell cycle to enable recovery from radiation-induced myelosuppression. A combination of gene expression and genetic strategies reveal that this aberrant response is due in part to elevated expression of the cyclin-dependent kinase inhibitor p21, although other factors are also likely involved.

Our studies reveal a differential capacity for $p 53^{7 K R}$ to regulate a subset of target genes in subtypes of cells in the hematopoietic system following genotoxic stress. Our data show that p53-induced proapoptotic genes, including puma, bax, noxa, and apf1, are expressed at relatively high levels in fully differentiated cells (thymocytes and splenocytes) after irradiation. In contrast, genes that are involved in the cell cycle, such as $p 21, p t e n$, and $f b x w 7$, are highly expressed in hematopoietic stem or progenitor cells (BM and Lin $^{-}$cells) after irradiation (Fig. 5). The ability of p53 to differentially regulate genes is likely to be dependent on additional factors such as its binding affinity to the distinct p53 response elements in each promoter as well as promoter chromatin structure, binding of other regulatory proteins, availability of cofactors, etc. (for review, see Espinosa 2008). Here, we provide the first in vivo evidence that modifications in the highly conserved C-terminal regulatory domain contribute to differential, tissue-specific gene regulation. The subset of genes that were altered by the $7 \mathrm{KR}$ mutations was distinct from that identified in $M d m 2^{+/-}$cells (Fig. 6C; Supplemental Fig. S13), suggesting that induction of different $\mathrm{p} 53$ response programs can be regulated by $\mathrm{C}$-terminal modifications. The importance of this region in p53 transcriptional control is consistent with structural studies indicating that the loose, positively charged residues within this highly conserved regulatory domain facilitate target gene identification by enabling p53 to encircle and slide along DNA (Jayaraman and Prives 1995; Weinberg et al. 2004; Tidow et al. 2007). Our data demonstrate that $p 21$ is one of the most exquisitely sensitive p53 response genes affected by p53 C-terminal modification in hematopoietic cells. Interestingly, DNA 
sequence and biochemical analyses indicate that the p21 gene contains one of the strongest p53-binding sites (Inga et al. 2002), and the gene may be poised to express rapidly due to both a special configuration of the transcriptional apparatus and the presence of preloaded polymerase II (Espinosa et al. 2003). Together, such factors likely enable p21 to manifest significant changes in the face of subtle alterations in p53 abundance or binding. It is possible that other genes that are hyperresponsive to $\mathrm{p} 53^{7 \mathrm{KR}}$ exhibit similar properties.

Control of p53 abundance and activity are clearly critical for survival and response to oncogenic and genotoxic stresses (Wade et al. 2010). Mdm2 plays key roles in both levels of control by virtue of its ubiquitin ligase activity and its ability to bind to the $\mathrm{N}$-terminal TAD and inhibit p53-dependent effects on transcription. Our data clearly show that preventing C-terminal lysine modifications targeted by Mdm2 for ubiquitylation is apparently not sufficient to completely stabilize p53, as p53 ${ }^{7 \mathrm{KR}}$ levels still increase after irradiation (Fig. 5D). This may be due to the use of alternative ubiquitylation sites, as indicated by our previous study showing that $\mathrm{p} 53^{7 \mathrm{KR}}$ is aberrantly ubiquitylated (Krummel et al. 2005). However, the activity of the $p 53^{7 K R}$ mutant is still exquisitely sensitive to $\mathrm{Mdm} 2$, as removing only one $M d m 2$ allele significantly increased radiosensitivity of $p 53^{7 K R}$ mice but not $p 53^{W T}$ mice in response to $5 \mathrm{~Gy}$ irradiation (Fig. 5A). Given this result, we were surprised to find that p53 levels do not strictly correlate with $\mathrm{Mdm} 2$ in the case of $p 53^{7 K R}$ animals. Thus, while p53 ${ }^{\mathrm{WT}}$ increased about twofold, at baseline, in $M d m 2^{+/-}$animals, $p 53^{7 K R}$ levels were, if anything, slightly less abundant in $p 53^{7 K R} ; \mathrm{Mdm}^{+/-}$than $p 53^{7 K R} ; M d m 2^{+/+}$animals at baseline and after irradiation (Fig. 5D, lanes 5-8). Interestingly, Mendrysa et al. 2003, 2006) have made similar observations using mice that express $\sim 30 \%$ of the WT level of Mdm2. In their analyses, the thymus in $M d m 2^{\text {puro/47-12 }}$ mice was smaller, and they observed increased p53 activity with similar to lower p53 levels. They also reported that the mutant thymus and spleen contained reduced numbers of leukocytes, indicating how p53 activity can affect cellular composition in these organs. Furthermore, their analyses showed significant decreases of WBCs in $M d m 2^{\text {puro/47-12 }}$ mice than $M d m 2^{\text {puro/+ }}$ and $M d m 2^{+/-}$mice. These results are similar to what we observed in $p 53^{7 K R} ; M d m 2^{+/-}, p 53^{7 K R}$, and $M d \mathrm{~m}^{+/-}$mice (Fig. 5B; Supplemental Fig. S7). Thus, the altered cellular composition in the $p 53^{7 K R} ; M d m 2^{+/-}$ spleens might contribute to p53 levels that are significantly different from WT, especially when employing methods such as Western blotting that detect protein levels in mixed cell populations. It is also possible that $p 53^{7 K R} ; M d m 2^{+/-}$cells with low p53 levels were positively selected through development. While the exact mechanism underlying the observed difference requires further investigation, it seems even at WT levels of expression, the $\mathrm{C}$ terminally altered $\mathrm{p} 53^{7 \mathrm{KR}}$ protein is more efficient than $\mathrm{p} 53^{\mathrm{WT}}$ at regulating a subset of genes in BM, such as $p 21$.

Radiosensitivity in the hematopoietic system is determined by the severity of irradiation-induced apoptosis in mature blood cells during the acute phase, and by the ability of HSCs and progenitors to repopulate and replace the damaged cells during the recovery phase. The proapoptotic gene puma and cell cycle arrest gene $p 21$ are two well-studied p53-inducible genes reported previously to contribute to radiosensitivity and HSC regulation. While puma deficiency protects HSCs from high-dose radiationinduced damage (Shao et al. 2010; Yu et al. 2010), the role of p21 in HSC regulation remains controversial owing to conflicting reports that the expanded HSC pool in p21deficient mice is exhausted over time due to inappropriate cell cycle entry (Cheng et al. 2000; van Os et al. 2007). Other studies reporting increased radiosensitivity in Atmand p21-deficient mice (Wang et al. 1997; Ito et al. 2007) suggest that the loss of cell cycle control in such animals generates defects in G1/S boundary control that could result in unscheduled S-phase entry and p53-dependent and -independent death processes. On the other hand, overexpressing $p 21$, which was observed in irradiated $p 53^{7 K R} \mathrm{BM}$, could result in a significant number of HSCs or progenitor cells undergoing cell cycle arrest. This could, in turn, cause a delay or insufficient repopulation of hematopoietic cells during the recovery phase after irradiation or other forms of genotoxic myelosuppression. Consistent with this, we found significantly less BrdU incorporation in irradiated $p 53^{7 K R}$ HSC and progenitor compartments (Fig. 6E) and low BM cellularity 2 and 4 wk after irradiation (Figs. 2D; Supplemental Fig. S2). Intriguingly, we also observed greater BrdU incorporation in $p 53^{7 K R}$ total BM-MNCs relative to WT during homeostasis (Fig. 6E). Since $p 53^{7 K R}$ mice contain similar numbers of total BM cells at baseline (Fig. 2D), it is conceivable that the reduced HSC frequency in $p 53^{7 K R}$ mice (Fig. 4A,B) might trigger a positive compensating stimulus that results in more progenitor or terminally differentiated cells entering cell cycle to generate sufficient mature cells to sustain hematopoiesis (Jankovic et al. 2007; Madan et al. 2009). In our study, reducing p 21 levels by losing one allele of $p 21$ completely rescued radiosensitivity of $p 53^{7 K R}$ mice at $5 \mathrm{~Gy}$, and partially rescued at $6 \mathrm{~Gy}$ of $\gamma$-irradiation, suggesting that complete restoration of HSC function in $p 53^{7 K R}$ mice after irradiation likely requires additional factors. While puma and other proapoptotic genes clearly contribute to p53-dependent radiosensitivity, such genes were not differentially up-regulated in the $p 53^{7 K R}$ mutant (Fig. 6C), raising the possibility that other genes that are expressed at greater levels in the mutant may contribute to its radiosensitive phenotype. Candidates for genes include those in Figure 6C that are highly up-regulated in the $p 53^{7 K R}$ mutant (highlighted in pink).

We also observed that $p 53^{7 K R} \mathrm{BM}$ exhibited extremely low repopulating efficiency in the competitive BM transplant assay even in the absence of irradiation. It is possible that transplant-associated stress (Lahav et al. 2005) cooperates with the elevated basal p53 activation of $p 53^{7 K R}$ HSCs and progenitors to produce the observed low repopulating efficiency of nonirradiated $p 53^{7 K R}$ BM (Fig. 3). A homing defect preventing $p 53^{7 K R}$ HSCs from accessing the hematopoietic niche could also contribute to their low repopulating efficiency. We have no direct evidence for such a deficiency, and note that $p 53^{7 K R}$ cells were present 
in both circulating peripheral blood and BM 6 and $12 \mathrm{wk}$ after transplant. However, we cannot rule out the possibility that $p 53^{7 K R}$ cells might exhibit different homing kinetics, which causes a delay in HSC niche engraftment. Recently, p53 activity level has been demonstrated to be a critical determinant of the ability of stem cells to compete in the setting of BM transplantation (Bondar and Medzhitov 2010; Marusyk et al. 2010). Similar to our observations, Bondar and Medzhitov (2010) found that cell cycle regulation plays an important role in p53dependent HSC competition. Our data add a mechanistic function to explain roles for p53 in transplantation competitiveness by showing that p21 levels can profoundly influence HSC/progenitor fitness, and that the p53 Cterminal regulatory domain appears to be one element at which signals elicited during transplantation are processed. This competition model implies that cells with compromised p53 function may gain selective advantages and contribute to tumor development or evolution.

Together, our and other studies (Mendrysa et al. 2003; Terzian et al. 2007; Wang et al. 2009) reveal the exquisite sensitivity of the hematopoietic system to modest elevations or reductions in p53 activity. By extension, transient antagonism of p53 activity could be protective in situations where environmental or chemotherapeutic genotoxins would otherwise be cytotoxic in normal tissues and stem cells (Gudkov and Komarova 2007). Furthermore, we and others previously showed that suppressing p53 activity enhances reprogramming of differentiated cells to pluripotent stem cells (Kawamura et al. 2009; Krizhanovsky and Lowe 2009). An implication of the data shown here is that transient p53 antagonism could also be beneficial for in vitro stem cell culturing to prevent stressinduced stem cell senescence, as BM transplants often require HSC enrichment or expansion ex vivo. In vivo, inappropriate repression of p53 C-terminal acetylation in PML-RAR oncoprotein-associated acute promyelocytic leukemia (APL) (Insinga et al. 2004) may compromise p53 activity sufficiently to enable rare leukemic stem cells to self-renew, generate leukemic blasts, and fuel leukemia progression. Interestingly, acute leukemias rarely contain p53 mutations but do exhibit compromised p53 function (Dino et al. 1994). The enhanced understanding of p53 regulation in HSC homeostasis and recovery from genotoxic injury afforded by these analyses suggest routes to the development of therapeutic approaches to spare or protect the hematopoietic system.

\section{Materials and methods}

\section{Mice and irradiation procedure}

C57Bl/6-Ly5.2 and C57Bl/6-Ly5.1 mice were purchased from Taconic. p53 ${ }^{7 K R}$ mice were generated previously (Krummel et al. $2005)$ and backcrossed to $\mathrm{C} 57 \mathrm{Bl} / 6$ background. $p 53^{7 K R}$ mice that were used in the study are in B6 or a mixed B6/129 background. $\mathrm{Mdm} 2^{+/-}$mice (kind gift of G. Lozano) are in a mixed B6/129 background. The $\mathrm{p} 21^{+/-}$mice used here (K Tinkum, D PiwnicaWorms, and H Piwnica-Worms, in prep.) are in a mixed B6/129 background; Supplemental Figure S14 shows that this p21 knockout allele expresses no detectable p21 following irradiation. For the irradiation study, age- and gender-matched mice were exposed to whole-body irradiation with a ${ }^{60} \mathrm{Co} \gamma$-irradiator at an average rate of $0.5 \mathrm{~Gy} / \mathrm{min}$. All mice used in the study were between 8 and 12 wk of age. All mouse maintenance and procedures were approved by the Animal Care and Use Committee of the Salk Institute.

\section{Histochemical analysis}

Tissues were isolated from mice and fixed in $10 \%$ neutral buffered formalin (Protocol) for $24 \mathrm{~h}$. Bones were decalcified in Cal-Ex solution (Thermo-Fisher). Fixed tissues were embedding in paraffin. Paraffin sections were deparaffinized and heated in 10 $\mathrm{mM}$ citrate buffer $(\mathrm{pH}$ 6) in a microwave for antigen retrieval. The sections were then stained with H\&E for pathological analysis. Apoptosis was determined by TUNEL assay using In Situ Cell Death Detection kit, Fluorescein (Roche).

\section{Colony-forming cell assay}

BM cells were flushed from femurs and tibias using IMDM medium (Gibco) containing 2\%FBS. Cells were triturated and filtered through a nylon screen to obtain a single-cell suspension. An aliquot of cells were stained with Turk solution for MNC counting. BM-MNCs $\left(5 \times 10^{5} \sim 5 \times 10^{6}\right)$ were plated for colonyforming cell assay using MethoCult and MegaCult-C (Stem Cell Technologies) following the manufacturer's instructions. Cells were incubated at $37^{\circ} \mathrm{C}$ with $5 \% \mathrm{CO}_{2}$ and $>95 \%$ humidity. Colonies of CFU-GM and BFU-E were scored on day 7 and colonies of CFU-Mk were scored on day 12.

\section{Competitive long-term reconstitution assay}

Donor BM-MNCs were isolated from WT or $p 53^{7 K R}$ mice $(\mathrm{C} 57 \mathrm{Bl} /$ 6-Ly5.2) and mixed with $2 \times 10^{5}$ competitive BM-MNCS isolated from congenic $\mathrm{C} 57 \mathrm{Bl} / 6$-Ly5.1 mice. The mixture was retroorbitally injected into lethally irradiated recipients (C57Bl/6Ly5.1). Twelve weeks post-transplants, reconstitution of donor leukocytes were analyzed by staining blood cells or BM with antibodies against leukocyte cell surface markers CD45.1 and CD45.2 (BD Pharmingen) using flow cytometry.

\section{Western blot analysis}

Cells or tissues were lysed in RIPA buffer $(50 \mathrm{mM}$ Tris- $\mathrm{HCl}$ at $\mathrm{pH}$ 7.4, $150 \mathrm{mM} \mathrm{NaCl}, 0.1 \%$ SDS, $0.5 \%$ sodium deoxycholate, $1 \%$ NP40, $10 \mathrm{mM} \mathrm{Na}_{3} \mathrm{VO}_{4}, 100 \mathrm{mM} \mathrm{NaF}$, protease inhibitor cocktails [complete tablet, Roche]). Protein extracts were analyzed by Western blotting using antibodies specific for p53 (1C12, Cell Signaling), p21 (BD Pharmigen), and Actin (Sigma).

\section{Real-time RT-PCR}

Total RNA from thymus or BM was isolated using RNeasy mini kit (Qiagen) and subjected to real-time qPCR as described previously (Krummel et al. 2005).

\section{Microfluidic $q P C R$}

Total RNA from thymus or BM was isolated using an RNeasy mini kit (Qiagen). The first strand cDNA was synthesized using SuperScript III kit (Invitrogen) followed by specific target amplification using primer pair sets specific for $\mathrm{p} 53$-induced genes and hprt and gapdh as controls. Preamplified gene expression assays were provided by Fluidigm Assay Design. Specific target amplification PCR products were loaded onto a 48.48 Dynamic Array Integrated Fluidic Circuits (Fluidigm), and qPCR thermal cycling was performed using a BioMark instrument (Fluidigm) following 
the manufacturer's instructions. Data were analyzed using Fluidigm Melting Curve Analysis software (Fluidigm). Expression of p53induced genes was normalized to the average signals of control genes hprt and gapdh, and relative expression of each gene from all the samples was scaled from 0 to 1 . Hierarchical clustering was performed using MeV 4.4 software (TM4 microarray software suite).

\section{Flow cytometry}

Antibodies are purchased from BD Pharmingen or mentioned elsewhere. BM cells were incubated with FITC or biotinconjugated antibodies against lineage markers including B220, CD3, Gr1, Mac1, Ter119, biotin, or PerCP-Cy5.5-conjugated anti-c-Kit; PE- or APC-conjugated anti-Scal (Biolegend); APCCy7-conjugated stretavidin; FITC-conjugated anti-CD41 and anti-CD48; PE-conjugated anti-CD150 (Biolegend), and FITCconjugated anti-CD34 (eBioscience). Cells were analyzed using FACScan, LSRI, or LSRII (Becton-Dickinson), and data were analyzed using CellQuest Pro and FlowJo software.

\section{BrdU incorporation}

Mice were intraperitoneally injected with $2 \mathrm{mg}$ of BrdU (Sigma) followed by the administration of $\operatorname{BrdU}(1 \mathrm{mg} / \mathrm{mL})$ in the drinking water for $2 \mathrm{~d}$. Mice were sacrificed and BM cells were isolated for the analysis of BrdU incorporation using APC BrdU flow kit (BD Biosciences).

\section{Statistical analysis}

We used the two-way $t$-test for analyzing differences between two groups. Data are represented as mean \pm SEM unless otherwise noted, with values of $P<0.05$ considered significant.

\section{Acknowledgments}

We thank Daphne Chen and Daniel Kim for mouse colony assistance and BM extraction, Dr. Grant Barish for the help with BM transplantation, and Rose Rodewald for technical assistance. We thank Dr. Alain Mir from Fluidigm Corporation for designing primers and his assistance in microfluidic chip analysis. This work was supported by grants from NCI (grants CA100845 and CA61449 to G.M.W., and CA094056 to D.P.W.) and the Cancer Center Core Grant for Core Facility support (grant 5 P30 CA014195). A.B. and J.-H.M. acknowledge support from the NCI (U01 CA84244) and the DOE Low Dose Program.

\section{References}

Appella E, Anderson CW. 2001. Post-translational modifications and activation of p53 by genotoxic stresses. Eur I Biochem 268: $2764-2772$.

Aranda-Anzaldo A, Dent MAR. 2007. Reassessing the role of p53 in cancer and ageing from an evolutionary perspective. Mech Ageing Dev 128: 293-302.

Bondar T, Medzhitov R. 2010. p53-mediated hematopoietic stem and progenitor cell competition. Cell Stem Cell 6: 309-322.

Chen J, Felicia ME, Keyvan K, Stephanie OO, Marie JD, Michael AE, Neal SY. 2008. Enrichment of hematopoietic stem cells with SLAM and LSK markers for the detection of hematopoietic stem cell function in normal and Trp53 null mice. Exp Hematol 36: 1236-1243.

Cheng T, Rodrigues N, Shen H, Yang Y-g, Dombkowski D, Sykes M, Scadden DT. 2000. Hematopoietic stem cell quiescence maintained by p21cip1/waf1. Science 287: 1804-1808.
Dino T, Letizia L, Andrea B, Lilla C, Rossella C, Fausto G, Anna Teresa M, Pier Giuseppe P, Antonino N. 1994. Analysis of p53 gene mutations in acute myeloid leukemia. Am $J$ Hematol 46: 304-309.

Espinosa JM. 2008. Mechanisms of regulatory diversity within the p53 transcriptional network. Oncogene 27: 4013-4023.

Espinosa JM, Verdun RE, Emerson BM. 2003. p53 Functions through stress- and promoter-specific recruitment of transcription initiation components before and after DNA damage. Mol Cell 12: 1015-1027.

Fauci A, Braunwald E, Martin JB. 2008. Harrison's principles of internal medicine. McGraw-Hill Companies, New York.

Feng L, Lin T, Uranishi H, Gu W, Xu Y. 2005. Functional analysis of the roles of posttranslational modifications at the p53 $\mathrm{C}$ terminus in regulating p53 stability and activity. Mol Cell Biol 25: 5389-5395.

García-Cao I, García-Cao M, Martín-Caballero J, Criado L, Klatt P, Flores J, Weill J, Blasco M, Serrano M. 2002. 'Super p53' mice exhibit enhanced DNA damage response, are tumor resistant and age normally. EMBO 21: 6225-6235.

Gudkov AV, Komarova EA. 2003. The role of p53 in determining sensitivity to radiotherapy. Nat Rev Cancer 3: 117-129.

Gudkov AV, Komarova EA. 2007. Dangerous habits of a security guard: the two faces of p53 as a drug target. Hum Mol Genet 16: R67-R72. doi: 10.1093/hmg/ddm052.

Inga A, Storici F, Darden TA, Resnick MA. 2002. Differential transactivation by the p53 transcription factor is highly dependent on p53 level and promoter target sequence. Mol Cell Biol 22: 8612-8625.

Insinga A, Monestiroli S, Ronzoni S, Carbone R, Pearson M, Pruneri G, Viale G, Appella E, Pelicci P, Minucci S. 2004. Impairment of p53 acetylation, stability and function by an oncogenic transcription factor. EMBO 23: 1144-1154.

Ito K, Takubo K, Arai F, Satoh H, Matsuoka S, Ohmura M, Naka K, Azuma M, Miyamoto K, Hosokawa K, et al. 2007. Regulation of reactive oxygen species by Atm is essential for proper response to DNA double-strand breaks in lymphocytes. I Immunol 178: 103-110.

Jankovic V, Ciarrocchi A, Boccuni P, DeBlasio T, Benezra R, Nimer SD. 2007. Id1 restrains myeloid commitment, maintaining the self-renewal capacity of hematopoietic stem cells. Proc Natl Acad Sci 104: 1260-1265.

Jayaraman L, Prives C. 1995. Activation of p53 sequencespecific DNA bindingby short single strands of DNA requires the p53 C-terminus. Cell 81: 1021-1029.

Kaushansky K, Fox N, Lin NL, Liles WC. 2002. Lineage-specific growth factors can compensate for stem and progenitor cell deficiencies at the postprogenitor cell level: an analysis of doubly TPO- and G-CSF receptor-deficient mice. Blood 99: 3573-3578.

Kawamura T, Suzuki J, Wang YV, Menendez S, Morera LB, Raya A, Wahl GM, Belmonte JCI. 2009. Linking the p53 tumour suppressor pathway to somatic cell reprogramming. Nature 460: 1140-1144.

Kemp CJ, Wheldon T, Balmain A. 1994. p53-deficient mice are extremely susceptible to radiation-induced tumorigenesis. Nat Genet 8: 66-69.

Kiel MJ, Yilmaz ÖH, Iwashita T, Yilmaz OH, Terhorst C, Morrison SJ. 2005. SLAM family receptors distinguish hematopoietic stem and progenitor cells and reveal endothelial niches for stem cells. Cell 121: 1109-1121.

Krizhanovsky V, Lowe SW. 2009. Stem cells: the promises and perils of p53. Nature 460: 1085-1086.

Krummel KA, Lee CJ, Toledo F, Wahl GM. 2005. The C-terminal lysines fine-tune P53 stress responses in a mouse model but 
are not required for stability control or transactivation. Proc Natl Acad Sci 102: 10188-10193.

Kruse J-P, Gu W. 2009. Modes of p53 regulation. Cell 137: 609622.

Lahav M, Uziel O, Kestenbaum M, Fraser A, Shapiro H, Radnay J, Szyper-Kravitz M, Avihai S, Hardan I, Shem-Tov N, et al. 2005. Nonmyeloablative conditioning does not prevent telomere shortening after allogeneic stem cell transplantation. Transplantation 80: 969-976.

Liu Y, Elf SE, Miyata Y, Sashida G, Liu Y, Huang G, Di Giandomenico S, Lee JM, Deblasio A, Menendez S, et al. 2009. p53 regulates hematopoietic stem cell quiescence. Cell Stem Cell 4: 37-48.

Madan V, Madan B, Brykczynska U, Zilbermann F, Hogeveen K, Dohner K, Dohner H, Weber O, Blum C, Rodewald H-R, et al. 2009. Impaired function of primitive hematopoietic cells in mice lacking the mixed-lineage-leukemia homolog Mll5. Blood 113: 1444-1454.

Marusyk A, Porter CC, Zaberezhnyy V, DeGregori J. 2010. Irradiation selects for p53-deficient hematopoietic progenitors. PLoS Biol 8: e1000324. doi: 10.1371/journal.pbio.1000324.

Mendrysa SM, McElwee MK, Michalowski J, O'Leary KA, Young KM, Perry ME. 2003. mdm2 is critical for inhibition of p53 during lymphopoiesis and the response to ionizing irradiation. Mol Cell Biol 23: 462-472.

Mendrysa SM, O'Leary KA, McElwee MK, Michalowski J, Eisenman RN, Powell DA, Perry ME. 2006. Tumor suppression and normal aging in mice with constitutively high p53 activity. Genes Dev 20: 16-21.

Meng A, Wang Y, Van Zant G, Zhou D. 2003. Ionizing radiation and busulfan induce premature senescence in murine bone marrow hematopoietic cells. Cancer Res 63: 5414-5419.

Rodriguez MS, Desterro JMP, Lain S, Lane DP, Hay RT. 2000. Multiple C-terminal lysine residues target p53 for ubiquitinproteasome-mediated degradation. Mol Cell Biol 20: 84588467.

Shao L, Sun Y, Zhang Z, Feng W, Gao Y, Cai Z, Wang ZZ, Look AT, Wu WS. 2010. Deletion of proapoptotic Puma selectively protects hematopoietic stem and progenitor cells against high-dose radiation. Blood 115: 4707-4714.

Tang Y, Luo J, Zhang W, Gu W. 2006. Tip60-dependent acetylation of p53 modulates the decision between cell-cycle arrest and apoptosis. Mol Cell 24: 827-839.

Tang Y, Zhao W, Chen Y, Zhao Y, Gu W. 2008. Acetylation is indispensable for p53 activation. Cell 133: 612-626.

TeKippe M, Harrison D, Chen J. 2003. Expansion of hematopoietic stem cell phenotype and activity in Trp53-null mice. Exp Hematol 31: 521-527.

Terzian T, Wang Y, Van Pelt CS, Box NF, Travis EL, Lozano G. 2007. Haploinsufficiency of Mdm2 and Mdm4 in tumorigenesis and development. Mol Cell Biol 27: 5479-5485.

Tidow H, Melero R, Mylonas E, Freund SMV, Grossmann JG, Carazo JM, Svergun DI, Valle M, Fersht AR. 2007. Quaternary structures of tumor suppressor p53 and a specific p53DNA complex. Proc Natl Acad Sci 104: 12324-12329.

Toledo F, Wahl GM. 2006. Regulating the p53 pathway: in vitro hypotheses, in vivo veritas. Nat Rev Cancer 6: 909-923.

Tyner SD, Venkatachalam S, Choi J, Jones S, Ghebranious N, Igelmann H, Lu X, Soron G, Cooper B, Brayton C, et al. 2002. p53 mutant mice that display early ageing-associated phenotypes. Nature 415: 45-53.

Uchida N, Aguila HL, Fleming WH, Jerabek L, Weissman IL. 1994. Rapid and sustained hematopoietic recovery in lethally irradiated mice transplanted with purified Thy1.1lo Lin-Sca- ${ }^{+}$hematopoietic stem cells. Blood 83: 3758 3779 . van Os R, Kamminga LM, Ausema A, Bystrykh LV, Draijer DP, van Pelt K, Dontje B, de Haan G. 2007. A limited role for p21Cip1/Waf1 in maintaining normal hematopoietic stem cell functioning. Stem Cells 25: 836-843.

Vousden KH, Prives C. 2009. Blinded by the light: the growing complexity of p53. Cell 137: 413-431.

Wade M, Wang YV, Wahl GM. 2010. The p53 orchestra: Mdm2 and Mdmx set the tone. Trends Cell Biol 20: 299-309.

Wang YA, Elson A, Leder P. 1997. Loss of p21 increases sensitivity to ionizing radiation and delays the onset of lymphoma in atm-deficient mice. Proc Natl Acad Sci 94: 14590-14595.

Wang Y, Schulte BA, LaRue AC, Ogawa M, Zhou D. 2006. Total body irradiation selectively induces murine hematopoietic stem cell senescence. Blood 107: 358-366.

Wang YV, Leblanc M, Wade M, Jochemsen AG, Wahl GM. 2009. Increased radioresistance and accelerated B cell lymphomas in mice with Mdmx mutations that prevent modifications by DNA-damage-activated kinases. Cancer Cell 16: 33-43.

Weinberg RL, Freund SMV, Veprintsev DB, Bycroft M, Fersht AR. 2004. Regulation of DNA binding of p53 by its C-terminal domain. J Mol Biol 342: 801-811.

Wognum AW, Eaves AC, Thomas TE. 2003. Identification and isolation of hematopoietic stem cells. Arch Med Res 34: 461475.

Yu H, Shen H, Yuan Y, XuFeng R, Hu X, Garrison SP, Zhang L, Yu J, Zambetti GP, Cheng T. 2010. Deletion of Puma protects hematopoietic stem cells and confers long-term survival in response to high-dose $\gamma$-irradiation. Blood 115: 3472-3480. 


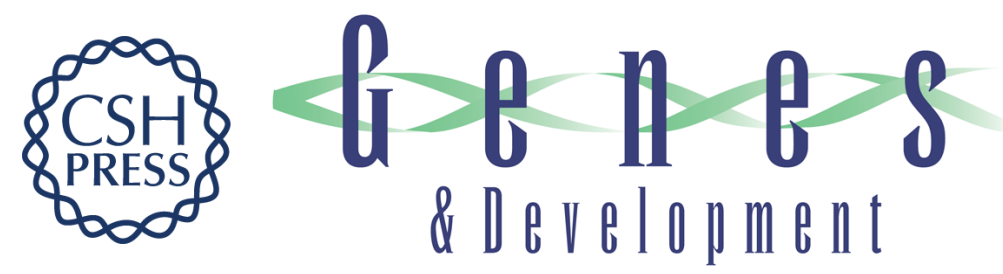

\section{Fine-tuning p53 activity through C-terminal modification significantly contributes to HSC homeostasis and mouse radiosensitivity}

Yunyuan V. Wang, Mathias Leblanc, Norma Fox, et al.

Genes Dev. 2011, 25:

Access the most recent version at doi:10.1101/gad.2024411

Supplemental http://genesdev.cshlp.org/content/suppl/2011/07/01/25.13.1426.DC1
Material

References This article cites 50 articles, 20 of which can be accessed free at:

http://genesdev.cshlp.org/content/25/13/1426.full.html\#ref-list-1

License

Email Alerting

Receive free email alerts when new articles cite this article - sign up in the box at the top

Service

right corner of the article or click here.

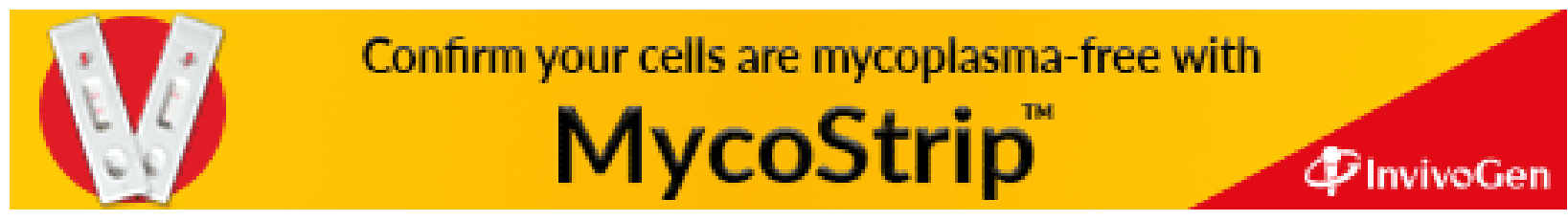

